\title{
Hidracáridos (Acari, Hydrachnidia) de la cuenca Andina del río Beni, Bolivia
}

\author{
Beatriz Rosso de Ferradás ${ }^{1}$ Hugo R. Fernández ${ }^{2} \&$ Blanca G. Rocabado ${ }^{3}$ \\ 1. Facultad de Ciencias Exactas, Físicas y Naturales, Universidad Nacional de Córdoba, Av. Vélez Sarsfield 299, 5000 Córdoba, \\ Argentina (CONICET). \\ 2. Facultad de Ciencias Naturales e Instituto Miguel Lillo, Universidad Nacional de Tucumán, Miguel Lillo 205, 4000 Tucumán, \\ Argentina (CONICET). \\ 3. Facultad de Ciencias Puras y Naturales, Universidad Mayor de San Andrés, Casilla 10077, La Paz, Bolivia.
}

\begin{abstract}
Hydracarids (Acari, Hydrachnidia) from the Andean basin of Beni river, Bolivia. Water mites from Andean part of the Bolivian Amazonian rivers were examined. A new species is described, Limnesia aymara. New records and redescription are made for Hygrobatella multiacetabulata Cook,1980, Atractides brasiliensis (Lundblad, 1937) and Krendowskia convexa (Ribaga, 1902). A new subgenus Schwoerbelobatella for hygrobatelids poliacetabulated is proposed and characterized. Ecological characteristics of the river area sampled and ecological preferences of the analyzed species are discussed.
\end{abstract}

KEYWORDS. New subgenus, tropical andean, Hydrachnidia, ecology.

\section{INTRODUCCIÓN}

En un primer aporte sobre la hidracarofauna del territorio boliviano se han registrado los taxa conocidos, descripto y redescriptos otros, así como sus ambientes (Fernández \& Rosso de Ferradás, 2001); posteriormente se suma la especie Thermacarus andinus Martin \& Schwoerbel, 2002 de los salares del suroeste de este país. A pesar de ser uno de los grupos hiperdiversos, los ácaros no han recibido la atención esperada en estudios de biodiversidad, especialmente en ecosistemas tropicales (Walter \& Proctor, 1998; Di Sabatino et al., 2000).

Los extensos y ricos recursos hídricos de Bolivia se diferencian en tres cuencas, la endorreica o del Altiplano, la del Amazonas, de donde proviene el material del presente estudio y la del Plata. La cuenca del Amazonas se origina en la Cordillera Oriental de los Andes, tiene ríos de gran caudal, cuyos afluentes atraviesan diferentes gradientes altitudinales, desde los $6000 \mathrm{~m}$, hasta la llanura beniana-pandina de menos de $300 \mathrm{~m}$. De la confluencia de tres ríos cuyas cuencas están enteramente en Bolivia, como el Mamoré, el Madre de Dios y el Beni, más el Iténez (que corre paralelo a la frontera de Bolivia y Brasil) nace el río Madera, uno de los afluentes principales del Amazonas (FernÁNDEZ \& R. DE FERRADÁs, 2001).

El objetivo es estudiar los ácaros acuáticos de tres hidro-ecoregiones diferentes, características de la gran diversidad geológica, geomorfológica y climática presentes en la cuenca andina del río Beni (RocABADO \& WASSON, 1999).

\section{MATERIAL Y MÉTODOS}

Los ríos Chamaleo y Suapi son afluentes de la cuenca del río Beni, principal tributario del río Madera (FERnÁNDEZ \& R. DE FERRADÁs, 2001). Dada la extensión del río Suapi se ubicaron dos estaciones de muestreo en su recorrido (fig. 1). El río Suapi en la zona de los Yungas (SUA) se encuentra a $1271 \mathrm{~m}$ de altitud, $16^{\circ} 06^{\prime} 68^{\prime \prime} \mathrm{S}$ - $67^{\circ} 47^{\prime} 14^{\prime}$ 'W, en la hidro-ecoregión de los Yungas altos, Bolivia (WASSON \& BARRÈRE, 1999) y se caracteriza por presentar valles profundos. Las precipitaciones varían entre 2000 y $3500 \mathrm{~mm}$ de promedio anual y el rango de temperatura fluctúa entre 12 y $15^{\circ} \mathrm{C}$ y velocidad promedio del río es no menor a $39 \mathrm{~cm} / \mathrm{s}$; la conductividad es de $21 \mu \mathrm{S} / \mathrm{cm}$ (Rocabado \& WaSSON, 1999).

El río Suapi en su porción subandina (SUS) se encuentra a $250 \mathrm{~m}$ de altitud, $14^{\circ} 50^{\prime} 07^{\prime \prime} \mathrm{S}-67^{\circ} 37^{\prime} 31^{\prime \prime} \mathrm{W}$ en la hidro-ecoregión del subandino bajo (WASSON \& BARRÈRE, 1999). Se caracteriza por presentar una alternancia de serranías y depresiones. Las precipitaciones llegan a los $2000 \mathrm{~mm}$ de promedio anual y las temperaturas están comprendidas entre 22 y $26^{\circ} \mathrm{C}$. La velocidad promedio en el río es de $39,24 \mathrm{~cm} / \mathrm{s}$ y la conductividad es de $147 \mu \mathrm{S} / \mathrm{cm}$ (ROCABADO \& WASSON, 1999).

La estación sobre el río Chamaleo (CHA) se encuentra a $370 \mathrm{~m}$ de altitud, $15^{\circ} 24^{\prime} 85^{\prime}$ "S-67 $34^{\circ} 71^{\prime \prime} \mathrm{W}$, en la hidro-ecoregión del Subandino alto (WASSON \& BARRÈRE, 1999) caracterizada por presentar serranías con valles paralelos al eje de la cordillera Oriental, siendo las rocas de arcillas y areniscas que atraviesa muy erosionables. Las precipitaciones están comprendidas entre los 1300 y $1700 \mathrm{~mm}$ y las temperaturas entre los 20 y $26^{\circ} \mathrm{C}$. La velocidad promedio en el río es de $38,95 \mathrm{~cm} / \mathrm{s}$; presenta altos valores de $\mathrm{Ca}^{++}(39 \mathrm{mg} / \mathrm{L})$ y una conductividad de $156 \mu \mathrm{S} / \mathrm{cm}$ (ROCABADO \& WASSON, 1999).

Las técnicas empleadas para las variables físicoquímicas están explicadas en RoCABADO \& WASSON (1999). Los hidracáridos fueron colectados con una red Suber de $900 \mathrm{~cm}^{2}$, fijándose las muestras en campo con formol al 4\%; luego fueron diafanizados, disecados, montados y medidos (en micras, $\mu \mathrm{m}$ ) según la metodología ya explicitada por R. DE FerRadÁs \& Fernández (2001); la terminología usada para mencionar las diferentes partes de la morfología de los hidracáridos es la propuesta por R. de FerRadÁs \& Fernández (1995). En la nueva especie descrita, además de los datos merísticos del holotipo 


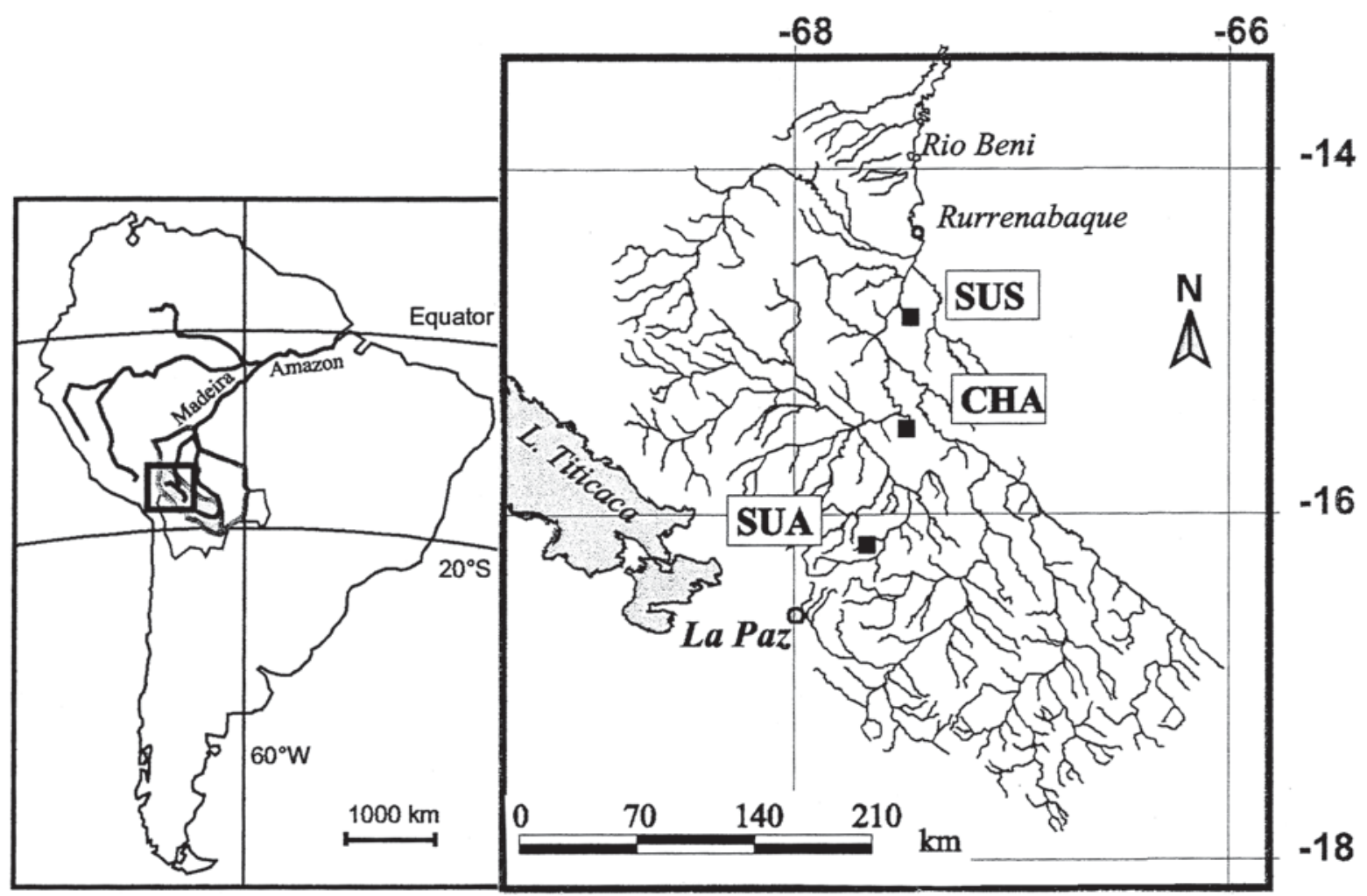

Fig. 1. Cuenca superior del río Beni, Bolivia y puntos de colectas: SUA, río Suapi (Yungas); SUS, río Suapi (subandino); CHA, río Chamaleo.

macho, en las hembras, figura en primer término las medidas del alotipo y entre paréntesis las de los paratipos.

Los ejemplares estudiados, se encuentran depositados en la Colección del Instituto-Fundación Miguel Lillo (IFML), Universidad Nacional de Tucumán, San Miguel de Tucumán, Argentina (Curador Dr. L. E. Grosso).

\section{RESULTADOS Y DISCUSIÓN}

Torrenticolidae

Torrenticola Piersig, 1896

Las especies del género tienen amplia distribución mundial, con excepción de Australia y gran parte de América del Sur, donde se las encuentran asociadas a ambientes del corredor andino. En América Central ha tenido una marcada especiación con más de 24 entidades (VIETS, 1977; COOK, 1980; CRAMER, 1992) con radiación hasta Colombia, donde se han encontrado seis especies y una subespecie, en los departamentos de Huila y Cauca (LuNDBLAD, 1953).

\section{Torrenticola (Torrenticola) columbiana (Lundblad, 1941)}

Atractides columbianus LundBLAD, 1941:103; localidad típica El Tambo, Colombia.

Torrenticola (s.str.) columbiana; LundBLAD, 1953:460.

T. (Torrenticola) columbiana; CoOK, 1980:46; R. DE Ferradás \& MATTONI, 1999:112; FernÁNDEZ et al., 2001:13.

Distribución. México y otras cuencas sudamericanas, relacionadas con los yungas de Argentina (СоОк, 1980; FernándeZ et al., 2001; extendiéndose hasta diversos limnótopos de las sierras de la província mediterránea de Córdoba, Argentina (R. de Ferradás \& Mattoni, 1999). Se registra en dos estaciones del río Suapi, Bolivia.

Material examinado. BOLIVIA, Río Suapi, Yungas altos, 2 f, 30.VI.1997, B. G. Rocabado (IFML A60050, A60051).

Comentario. Los especímenes estudiados no difieren morfológicamente de los cientos de ejemplares observados de Argentina, y sus dimensiones están comprendidas entre los amplios extremos de las dadas.

\section{Limnesiidae}

\section{Limnesia Koch, 1836}

Las especies de este género en Bolivia ya fueron comentados en trabajo anterior (FERNÁNDEZ \& R. DE FERRADÁs, 2001).

\section{Limnesia (Limnesia) aymara sp. nov. (Figs. 2-11)}

Holotipo macho. Cuerpo, forma oval: longitud, 1000; ancho, 750; platelet posterior para inserción muscular, subcuadrangular y porosa; diámetro, 57 ; ojos con dos pares de lentes redondeadas, proporcionalmente grandes; postocularias distanciadas de los ojos; cinco pares de dorso-glandularias (fig. 2); longitud del extremo anterior de las CX-I al borde posterior de las CX-IV, 467; ancho entre bordes externos de IV-pt, 603; glándula Limnesiae ubicada hacia el borde anterior de las Cx-III; el 
borde esclerosado de las Cx-III y IV sobrepasa la barra quitinizada media, cuyos bordes son ondulados, y se solapa con los bordes del grupo coxal anterior (fig. 3); CX-I de cada lado no anastomosadas; apodemas de las CX-I y II llegan al borde de las C-III; campo genital: longitud, 128; ancho, 126; bordeado con pelos largos; tres pares de acetabula con bordes ondulados; gonoporo alargado (fig. 7); epimeroglandularia 2 ubicada en línea vertical con respecto al pelo para-genital; capitulum levemente proyectado, con varios pares de púas, longitud ventral, 179; longitud dorsal de los segmentos palpales: P-I, 27; P-II, 132; P-III, 70; P-IV, 159; P-V, 58; pelos plumosos; el tubérculo ventral de P-II, en forma de clavija, corto y romo (fig. 4); extremo disto-ventral de P-IV con pequeña púa; longitud dorsal de los segmentos distales de las patas: I-pt-4, 111; I-pt-5, 127; I-pt-6, 132 (fig. 6); IVpt-4, 181; IV-pt-5, 189; IV-pt-6, 180, pelo final, 94; IV-pt-4 con 3 pelos natatorios; IV-pt-5 con 9 pelos natatorios; IV-pt-6 con 3 pelos simples proximales (fig. 5).

Alotipo hembra. Cuerpo redondeado: longitud, 1083 (1000); ancho, 771 (792); platelet, diámetro, 53; longitud entre el extremo anterior de $\mathrm{Cx}$-I al borde posterior de $\mathrm{Cx}$ IV, 480 (442-479); ancho entre los bordes externos de CxIV, 677 (636); campo genital: longitud, 169; con esclerito pre-genital, 197 (168-205); ancho máximo, 175 (140); hembra ovígera, huevos diámetro, 164 (173); acetabula con bordes ondulados, con un espacio marcado entre las anteriores y los dos pares posteriores; variado número de pelos en el borde medio de las valvas (figs. 8, 9); esclerito pre-genital grande y de bordes irregulares; quetotaxia y morfología de los palpos como en machos; longitud dorsal de los segmentos palpales: P-I, 29 (2331); P-II, 132 (148-137); P-III, 103 (93-105); P-IV, 184 (165171); P-V, 62 (58-60); capitulum, longitud ventral, 185; longitud dorsal de los segmentos distales de las patas: Ipt-4, 111 (117); I-pt-5, 132 (160); I-pt-6, 138 (128); IV-pt-4, 190 (189-188); IV-pt-5, 210 (169-182); IV-pt-6, 206, pelo distal, 90 (64-77); IV-pt-4 con 5 pelos natatorios; IV-pt-5 con 5 (8-10) pelos natatorios; IV-pt-6 con 3 (4) pelos cortos y plumosos proximales (figs. 10,11); patas primeras con fuertes pelos, sin pelos natatorios.

Diagnosis diferencial. Limnesia (Limnesia) aymara se diferencia de L. minuscula minuscula Ribaga, 1902 (sensu CooK, 1980) por, las Cx-IV, con borde interno recto; la esclerotización de las Cx-III y IV, llega al borde del grupo coxal anterior; las epimeroglandularia 2 y pelo paragenital están ubicados en línea vertical a partir de la mitad anterior del campo genital y próximo a éste; acetabula de bordes ondulados; P-II con clavija proporcionalmente corta y roma; IV-pt-5 con 9 pelos natatorios. En L. m. minuscula, las $\mathrm{Cx}-\mathrm{IV}$ tienen una amplia escotadura curva; la esclerotización de las Cx-III y IV no llega al grupo coxal anterior; las epimeroglandularia 2 y pelo para-genital ubicados en línea casi horizontal y en la parte posterior del campo genital; acetabula de bordes lisos; P-II con clavija larga y afinada y en IV-pt-5 lleva entre 4-5 pelos natatorios. De las subespecies de L. minuscula existen dos más afines, $L$. $m$. valida Lundblad, 1941 y L. $m$. distinta Viets, 1954. De L. m. valida, se separa netamente pues sus machos tienen las $\mathrm{Cx}$-I anastomosadas y los acetabula proporcionalmente grandes, ocupando toda la superficie del campo genital, tanto en machos como en hembras; P-
II con clavija y base pequeñas y todos los pelos de los palpos muy cortos; IV-pt-6 con 13 pelos natatorios y IVpt-5 con 16. Se diferencia asímismo, de L. m. distinta pues ésta tiene las $\mathrm{CX}$-I de cada lado anastomosadas en el centro, en tanto que las Cx-IV son proporcionalmente anchas; las epimeroglandularias 2 y pelo para-genital ubicados en una línea casi horizontal. Otras especies de Limnesia (Limnesia) morfológicamente muy próximas a L. aymara son L. sutava Cook, 1980, sólo conocidas las hembras, y $L$. braytola Cook, 1980. Las hembras de $L$. sutava tienen los apodemas del grupo coxal primero muy anchos y cortos, no llegan al borde de las Cx-III; esclerito pre-genital pequeño; sin pelo largo y plumoso en la superficie interna de P-III. Limnesia braytola tiene, en machos y hembras, una barra quitinizada en el extremo de las Cx-III de forma muy peculiar, ensanchada en sus ángulos extremos; los acetabula tienen sus bordes lisos y la epimeroglandularia 2 y pelos están ubicados en una línea oblicua y a la altura de la mitad posterior del campo genital.

Comentarios. Como la descripción original de Limnesia minuscula Ribaga, 1902, es incompleta e imprecisa y hay varias redescripciones, agregándose además, varias subespecies propuestas posteriormente (algunas no vigentes en la actualidad), nos hemos referido en la diagnosis, a la redescripción de Соок (1980), que es la más precisa y detallada.

El "grupo L. minuscula" está integrado por diferentes poblaciones, cuyas variaciones intra e intersubespecificas y status consiguientes son confusos. A partir de la descripción original y redescripción de $L$. minuscula (localidad típica Buenos Aires, Argentina, sin mayor precisión), se estableció un grupo de subespecies, no teniendo un morfotipo muy definido, solapándose sus caracteres así como las geonemias respectivas, que además responden a hallazgos muy limitados (RibaGA, 1902, 1903). Las subespecies propuestas fueron: $L .(L$. minuscula adulterina Lundblad, 1930, localidad típica Quebrada Roque, Perú; L.(L.) m. invalida Lundblad, 1941, de Arroyo Itá, Paraguay; L.(L.) m. valida Lundblad, 1941, Rio de Janeiro, Brasil; L.(L.) m. peruana (Lundblad, 1930), Quebrada Roque, Perú; $L$.(L.) m. distorta Viets, 1954, río Beberibe, Olinda, Pernambuco, Brasil. VIETs (1955) adjudica con algunas dudas los especímenes que estudió del lago Titicaca, Perú, a $L .(L$.$) m. minuscula y los de las$ cuencas lóticas ubicadas al oeste del lago a $L$. $(L$.) $m$. peruana (Lundblad, 1930). BesCH (1963), sinonimizó las subespecies $L$.(L.) m. invalida y $L$. $(L$.) $m$. peruana con $L$. $(L$.) m. minuscula, incluyendo además en esta sinonimia a $L .(L$.$) patagonica Lundblad, 1941. Este último hecho$ taxonómico fue refutado en trabajos posteriores, donde se estudiaron poblaciones de esta especie de diferentes cuencas patagónicas argentinas (СоOK, 1980; R. DE FERRADÁs et al.,1987; SMIT, 2002).

En el catálogo de VIETS (1987) se reconocen como válidas las subespecies $L(L$.) m. minuscula, $L$. ( $L$.) $m$. valida y $L .(L$. $) m$. distorta, así como $L$. patagonica. $\mathrm{La}$ corología de L. sutava Cook, 1980, está limitada a una localidad del río Metán, Salta, Argentina, en tanto que $L$. braytola Cook, 1980, ha sido registrada en ríos de Tucumán y Catamarca (Соок, 1980) y en limnótopos a diferentes alturas de las sierras de Córdoba, Argentina (R. DE FERRADÁs, 2000). 


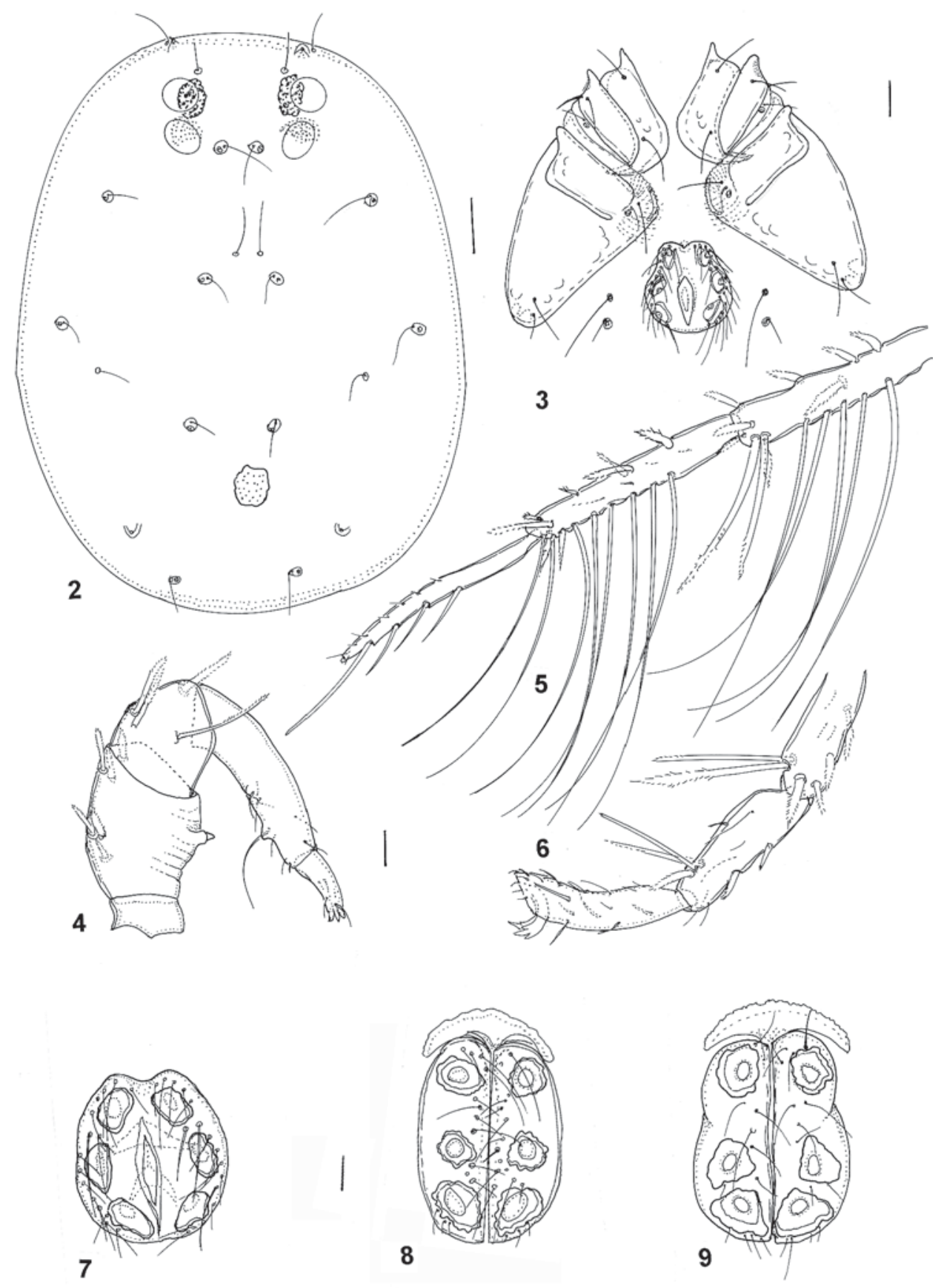

Figs.2-9. Limneisa aymara sp. nov., macho: 2, dorsal; 3, ventral; 4, palpo, vista interna; 5, IV-4-5, 6 de las patas 4; 6, I-pt-4-5, 6 de las patas $1^{\circ}$; 7, campo genital; hembra: 8,9 , ventral. Escalas: figs. 2, 3, $100 \mu \mathrm{m} ; 5,6,33 \mu \mathrm{m} ; 4,7,26 \mu \mathrm{m} ; 8,9,29 \mu \mathrm{m}$.

Material-tipo. Holotipo ơ, BOLIVIA, río Suapi, porción subandina, 13.X.1997, B. G. Rocabado col. (IFML A60060); alotipo $\mathcal{F}$, iguales datos de colección (IFML A60061); 4 paratipos c, iguales datos de colección (IFML A60062-65).

Etimología. El sustantivo específico en aposición es alusivo al grupo étnico Aymara, que poblaba los territorios montañosos de los alrededores del Titicaca.

\section{Hygrobatidae}

De esta familia se conocían para la hidracarofauna del territorio boliviano siete especies (FERNÁNDEZ \& R. DE FERRADÁs, 2001) sumándose ahora el registro de dos géneros y dos especies.

\section{Atractides Koch, 1837}

El género está representado en América del Sur por un grupo de especies relacionadas con reótopos, tanto brasílicos como andinos, siendo ésta la primera cita de una especie para el territorio de Bolivia.

\section{Atractides (Atractides) brasiliensis (Lundblad, 1937)}

Sinonimia: R. DE FERRADÁs \& MATTONI (1999:116).

Comentarios. Se ha observado una hembra ovígera, cuya morfología coincide con lo expresado por CoOK (1980) y ampliado por R. DE FERRADÁs \& MATTONi (1999) así como 
sus dimensiones se hallan comprendidas entre los extremos registrados.

Material examinado. BOLIVIA, río Suapi, zona de los Yungas, f, 30.VI.1997, B. G. Rocabado col. (IFML A60052).

\section{Hygrobatella Viets, 1926}

Este género ha sido motivo, como tantos otros hygrobátidos, de sucesivos cambios en los subgéneros que comprende (CoOK, 1980; 1988). Esto se debe a la poca precisión en las definiciones de cada uno de ellos, particularmente en aquellos en cuyas especies típicas no se conocían machos y hembras. Por este motivo recomendamos nominar subgéneros solamente cuando hayan sido estudiados machos y hembras.

Соок (1974) había incluido en Hygrobatella tres subgéneros, Hygrobatella Viets, 1956, con tres pares de acetabula; Tetrahygrobatella Lundblad, 1953, con tres a cinco pares de acetabula y diferentes características en las líneas de sutura de las coxas y Hygrobatulus Viets, 1953, con seis o más pares de acetabula, sinonimizando además con éste, a Polyhygrobatella Lundblad, 1953. Posteriormente Соок (1980) eleva el status de Tetrahygrobatella a género y se invalida a Hygrobatulus, pues su especie tipo, erigida en base a una hembra, pertenecía a una especie más antigua de otro género (Dodecabates Viets, 1926) quedando después de este acto nomenclatural, una especie de poliacetabulados (Hygrobatella polygramma Lundblad, 1953) y otra posible (sólo conocida por las hembras, $H$. multiacetabulata Cook, 1980) sin subgénero nominado que las contengan.

Dentro de Hygrobatella existen pues, de momento, dos grupos de especies netamente separados: uno, triacetabulados y otro multiacetabulados. El primer grupo, Hygrobatella (Hygrobatella) está integrado por 11 especies y seis subespecies, la mayoría de ellos ligados al corredor andino austral. El segundo, los multiacetabulados, está conformado por $H$. polygramma e $H$. multiacetabulata; la pertenencia de ésta última especie al grupo fue confirmada, con la descripción de los machos, por FERNÁNDEZ (1994). Se propone aquí un subgénero para este grupo innominado, ya bosquejado por Соок (1980).

\section{Schwoerbelobatella subgen. nov.}

Especie tipo: Hygrobatella (Polyhygrobatella) polygramma Lundblad, 1953, designación original. Typus, Prap. 3944, Colección Lundblad, Riksmuseum, Stockholm, Suecia.

Diagnosis. El número de acetabula del campo genital, no menos de 20 pares a cada lado del gonoporo, es definitorio para el grupo; otros aspectos morfológicos son compartidos con las especies del género, tales como: capitulum libre en ambos sexos; apodemas posteriores del primer grupo coxal extendido más allá del borde de las CX-III; sutura entre Cx-III y IV hasta las proximidades de la glandularia de la Cx-IV; palpos con pequeños dentículos en la superficie interno-ventral de P-II y III y seta distoventral en P-IV; patas sin marcado dimorfismo sexual.

Comentarios. Este subgénero contiene dos especies: Hygrobatella polygramma y $H$. multiacetabulata; en $H$. multiacetabulata, los palpos del macho tienen los segmentos P-IV y V, con respecto a la primer especie, proporcionalmente más largos, particularmente $\mathrm{P}-\mathrm{V}$, que es además, muy curvado y en posición distoventral de P-IV posee una seta espatulada, como así también, por un esclerito entre las coxas. Hygrobatella polygramma se la ha registrado solamente en una cuenca de los Andes colombianos.

Etimología. Este subgénero es un homenaje al Dr. Jürgen Schwoerbel.

\section{Hygrobatella (Schwoerbelobatella) multiacetabulata Cook 1980}

(Figs. 12-14)

Hygrobatella (?) multiacetabulata CooK, 1980:159 (localidad tipo, Río Metán, prov. Salta, Argentina); FERnáNDEZ, 1994: 221.

Macho. Cuerpo, forma oval: longitud, 834, ancho, 669; un par dorsalia pequeñas a la altura del tercer par de glandularia; longitud entre extremo anterior de la I-Cx y el borde posterior de la IV-Cx, 504; ancho a la altura Cx-IV, 619; longitud capitulum, 239; ventralia oval (fig.12) entre las IV-Cx, donde apoyan los músculos del extremo del capitulum (fig.14): largo, 82 y ancho, 53; placa genital de cada lado: longitud, 267, ancho, 218; longitud gonoporo, 86; plaquita poro excretor: longitud, 49; ancho, 33; longitud arista dorsal de los segmentos palpales: P-I, 53; P-II, 154; P-III, 140; P-IV, 220; P-V, 165; la seta del extremo distal de P-IV, espatuliforme (fig.13); longitud dorsal de los segmentos distales de las patas: I-pt-4, 273; I-pt-5, 281; I-pt-6, 206; IV-pt-4, 298; IV-pt-5, 413; IV-pt-6, 273.

Hembra. Cuerpo, oval: longitud, 925; ancho, 834; longitud entre extremo anterior de la I-Cx y el borde posterior de la IV-Cx, 586; ancho a la altura Cx-IV, 834; carece de ventralia; longitud capitulum, 264; mitad campo genital: longitud, 234; ancho, 189; longitud gonoporo (incluyendo escleritos genitales) 205; plaquita poro excretor: longitud, 37; ancho, 23; longitud arista dorsal de los segmentos palpales: P-I, 47; P-II, 148; P-III, 126, PIV, 195; P-V, 99; la seta del extremo distal de P-IV, espatuliforme (fig.14); longitud dorsal de los segmentos distales de las patas: I-pt-4, 223; I-pt-5, 227; I-pt-6, 198; IV-pt-4, 338; IV-pt-5, 421; IV-pt-6, 239.

Material examinado. BOLIVIA, río Suapi, zona de los Yungas, 2 o, 5 \&, 30.VI.1997, B. G. Rocabado col. (IFML AG0053-54, IFML AG0055-59, respectivamente); río Chamaleo, Subandino alto, 2 \&, 25.VII.1997, B. G. Rocabado col. (IFML AG0048-49).

Comentario. Es notable el desarrollo que alcanza P$\mathrm{V}$ en los machos y la densidad diferente de dentículos en la región ventral de P-II y P-III, entre ambos sexos (figs.13, 14). Como carácter dimórfico sexual, es destacable, la ventralia ubicada entre las IV-Cx en los machos, no señalada anteriormente. Los especímenes estudiados presentan además, una seta distal en P-IV, espatuliforme, no en forma de clavija como había sido señalado.

Las dos especies de este subgénero, están asociadas a la zona andina tropical a diferencia de las especies del subgénero Hygrobatella, que presentan un amplio rango de distribución latitudinal en América del Sur, si bien la mayoría de ellas han sido encontradas en limnótopos de los Andes australes. 

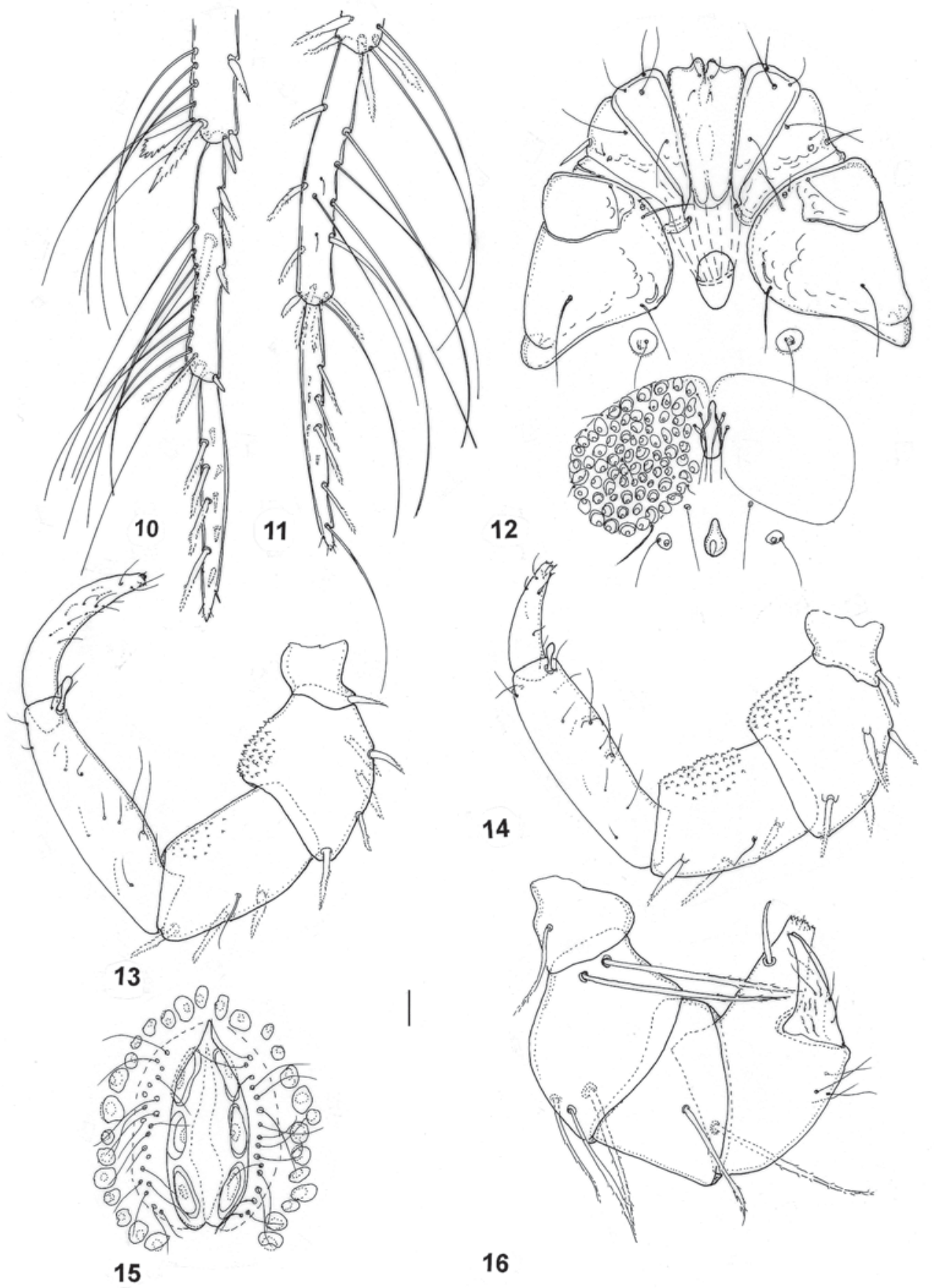

16

Figs.10-16. Limnesia aymara sp. nov. hembra: 10, IV-pt-4-5, 6 de las patas $4^{\circ}$; 11, IV-4-5, 6 de las patas $4^{\circ}$. Hygrobatella multiacetabulata Cook, 1980: 12, macho, vista ventral; 13, macho, palpo, vista interna; 14, hembra, palpo, vista interna. Krendowskia convexa (Ribaga, 1902) macho: 15, campo genital; 16, palpo, vista interna. Escalas: figs. 10, 11, $68 \mu \mathrm{m} ; 12,58 \mu \mathrm{m} ; 13,14,30 \mu \mathrm{m}$; $15,18 \mu \mathrm{m}$ y $16,46 \mu \mathrm{m}$.

\section{Krendowskiidae}

\section{Krendowskia Piersig, 1895}

Este género, si bien se han hallado algunas especies en otras partes del mundo (СоOK, 1974), alcanza su mayor riqueza en América del Sur con 10 especies (VIETS, 1987), nueve de ellas son brasílicas y sólo una se encuentra en limnótopos relacionados con las sierras pampeanas de Córdoba, Argentina (R. DE FerRADÁs, 1975) y Valparaíso, Chile (RIBAGA, 1902).
Krendowskia (Krendowskia) convexa (Ribaga, 1902)

(Figs. 15, 16)

Sinonimia: VIETS, 1987:421.

Machos. Escudos dorsal y ventral presentes; escudo dorsal: largo, 1260-1288; ancho, 882-952; entre las dorso-glandularias medias hay entre 14 y 15 poros; coxas: longitud entre el extremo anterior de las CX-I y el extremo posterior de las Cx-IV, 756-770; ancho, entre las apófisis articulares de las IV-Cx, 882-938; valvas genitales: longitud, 286-312; ancho, 259-299; tres pares de acetabula 
y abertura genital muy aguzada hacia adelante; longitud del gonoporo, 270-281; gonoporo rodeado de una desordenada hilera de pelos (fig.15); escotadura articular del capitulum en forma de V; capitulum, arista ventral, 205; longitud dorsal de los segmentos palpales: P-I, 4751; P-II, 103-105; P-III, 56-64; P-IV, 85-113; P-V, 61-70; todos los pelos de los palpos son pennados (con pocas y muy finas ramas laterales) el extremo disto-ventral de P-IV trunco y estriado (fig.16); longitud dorsal de los segmentos distales de las patas: I-pt-4, 201; I-pt-5, 238; Ipt-6, 201; II-pt-4, 242; II-pt-5, 284; II-pt-6, 234; III-pt-4, 242; III-pt-5, 271; III-6, 226; IV-pt-4, 255; IV-pt-5, 271; IVpt-6, 234; primer par de patas sin pelos natatorios; patas II a IV con número variable de pelos natatorios (nunca menos de 6); extremo distal de los segmentos con dientes de diferentes tamaño que sobrepasan la inserción del siguiente segmento.

Material examinado. BOLIVIA, río Chamaleo, Subandino alto, 2 ơ, 25.VIII.1997, B. G. Rocabado col. (IFML A60063$64)$.

Comentario. Para la determinación de este material se realizaron comparaciones con ejemplares de esta especie provenientes de dos lagos artificiales ubicados en las sierras pampeanas de la Provincia de Córdoba, Argentina, pues algunos caracteres como el patrón de coloración, en los especímenes provenientes de Bolivia, no pudo observarse dado que estos estaban fijados y conservados en formol. Otras cualidades como el gran tamaño de los especímenes; las estructuras de los palpos y su quetotaxia; el escudo ventral, coxas y campo genital tienen una total correspondencia con los especimenes de K. convexa estudiados por R. DE FERRADÁs (1975). Esta especie tiene una geonemia muy particular, habiéndosela encontrado en Valparaíso, Chile (sin explicitarse el tipo de cuenca) y en diferentes lagos artificiales ubicados en la sierras de la Provincia de Córdoba (Marshall, 1940; R. DE FERRADÁs, 1975) y en una pequeña laguna natural, con abundante hidrofitia, de la Pampa Olaen, sierras de Córdoba y en el cauce del río Chamaleo (Bolivia), también en condiciones no muy alejadas de los ambientes lénticos artificiales y naturales de Argentina. En el área intermedia (noroeste argentino) profusamente muestreada, aún no ha sido registrada esta especie.

Ecología. Las estaciones de las cuales provienen las muestras están ubicadas en la cuenca alta del río Beni (WASSON \& BARRÈRE, 1999) que abarca un gradiente altitudinal y espacial analizado por ROCABADO \& WASSON (1999). En él se usaron análisis multivariados para datos de abundancia de macroinvertebrados bentónicos y variables físico-químicas. Sus resultados mostraron que existe un importante gradiente físico en el primer eje (44\% de la variabilidad) debido a la pendiente, altura e inestabilidad del sustrato. Se puede decir que en el extremo izquierdo de ese eje se encuentran condiciones lóticas, mientras que en el derecho las condiciones son lénticas. En dicho análisis, los hidracáridos incluidos como tales no aportaron significativamente al ocupar una posición cercana al cruce de los ejes. Ahora, en un proceso inverso de recuperación de la información, a partir de la posición de las estaciones en el análisis citado y la identificación de las especies, se pueden hacer algunas generalizaciones sobre las preferencias ecológicas de estos ácaros acuáticos. Las especies aquí tratadas se distribuyen entre los dos extremos del gradiente físico encontrado por ROCABADO \& WASSON (1999). En el extremo lótico del gradiente con sustrato grueso, altura $>1000 \mathrm{~m}$, fuerte pendiente y temperatura $<25^{\circ} \mathrm{C}$ se encuentra a Torrenticola columbiana y Atractides brasiliensis. En el extremo léntico del gradiente con sustrato más fino, altura $<500 \mathrm{~m}$, temperatura $>25^{\circ} \mathrm{C}$ y sustrato inestable, se encuentra a Limnesia aymara. Entre ambos extremos, aunque más cercano a las características lénticas se ubica Krendowskia convexa. Hygrobatella multiacetabulata se distribuye prácticamente en ambos extremos del gradiente, mostrando al parecer una amplia tolerancia a los factores considerados. Esto estaría de acuerdo con la variedad de localidades donde fue colectada previamente en el noroeste de Argentina (СоOK, 1980; FernándeZ, 1994; FeRnándeZ, 2003). Estas especies responden en su distribución a las ya mencionadas características físicas del río. Algunas de ellas como la temperatura y la composición del sustrato ya fueron señaladas por otros autores y resumidas por Di SABATINO et al. (2000).

Agradecimientos. A I. González, de la Universidad Mayor de San Andrés, Bolivia, por proporcionarnos el mapa del área de estudio. Al CONICET (Consejo Nacional de Investigaciones Científicas y Técnicas), Universidad Nacional de Córdoba y Universidad Nacional de Tucumán, Argentina y a la Universidad Mayor de San Andrés, por el constante apoyo.

\section{REFERENCIAS BIBLIOGRÁFICAS}

Besch, W. 1963. Südandine Limnesiiden. Acarología, Paris, 5(2): 259-265.

Cook, D. 1974. Water mite genera and subgenera. Memoirs of the American Entomological Institute, Ann Arbor, 21: $1-860$.

1980. Studies on neotropical water mites. Memoirs of American Entomological Institute, Ann Arbor, 31:1645 .

1988. Water mites from Chile. Memoirs of the American Entomological Institute, Ann Arbor, 42:1-356.

Cramer, C. 1992. Estudios sobre hidracáridos mexicanos, familia Torrenticolidae. I. Cinco especies nuevas de Neoatractides y Torrenticola y primer registro de Testudacarus para México. Anales del Instituto de Biología, Universidad Nacional Autónoma de México, Serie Zoología, México, 63(1):13-27.

Di Sabatino, A.; Gerecke, R. \& Martin, P. 2000. The biology and ecology of lotic water mites (Hydrachnidia). Freshwater Biology, Oxford, 44:47-62.

Fernández, H. R. 1994. Notas sobre Hygrobatella multiacetabulata Cook (Hygrobatidae, Parasitengona, Acari). Acta Zoológica Lilloana, Tucumán, 43(1):221223 .

2003. Structure of water mite taxocoenoses in two northwestern Argentinean subtropical sub-catchments. Systematic and Applied Acarology, Auckland, 8:55-66.

Fernández, H. R. \& Rosso de Ferradás, B. 2001. Hidracáridos del altiplano boliviano: La cuenca alta del Río Suchez. Physis, Buenos Aires, Secc. B, 58(134-135):29-36.

Fernández, H. R.; Romero, V. F. et al. 2001. La diversidad del zoobentos en ríos de montaña del noroeste de Argentina: comparación entre seis ríos. Ecología Austral, Buenos Aires, 11:9-16.

LundBlad, O. 1941. Neue wassermilben. Vorlaufige Mitteilung. Entomoligisk Tidskrift, Stockholm, 92(1-2):97-121. 1953. Die hydracarinenfauna von Colombia. Arkiv för Zoologi, Uppsala, 5(8):435-585. 
Marshall, R. 1940. Water mites from Argentina. Transactions of American Microscopical Society, Lawrence, 59(3):377-382.

Ribaga, A. 1902. Acari sudamericani. Zoologischer Anzeiger, Jena, 25(675):502-508.

1903. Diagnosi di alcune specie nouve di Hydrachnidae e di un Ixodide del Sud America. Annali Scoula Superiore di Agricolture, Portici, 5(5):1-28.

Rocabado, G. \& Wasson, J. G. 1999. Regionalización de la fauna bentónica en la Cuenca Andina del río Beni (Bolivia). Revista Boliviana de Ecología, Cochabamba, 6:121132.

Rosso de Ferradás, B. 1975. Ácaros acuáticos (Acari, Hydrachnellae) de lagos de embalse y cuencas relacionadas de la província de Córdoba, República Argentina. Physis, Secc. B, Buenos Aires, 34(88):27-32.

__. 2000. Ácaros reófilos (Acari: Hydrachnidia) de las sierras de Córdoba. IV. Revista de la Sociedad Entomológica Argentina, Buenos Aires, 59(1-4):25-40.

Rosso de Ferradás, B. \& Fernández, H. R. 1995. Acari Hydrachnidia. In: Lopretto, E. \& Tell, G. Ecosistemas de aguas continentales. La Plata, Editorial Sur. p.819853.

2001. Acari. In: Fernández, H. R. \& Dominguez, E. Guía para la determinación de los artrópodos bentónicos Sudamericanos. San Miguel de Tucumán, Editorial Universitaria de Tucumán. p.237-256.

Rosso de Ferradás, B. \& Mattoni, C. I. 1999. Ácaros reófilos
(Acari: Hydrachnidia) de la sierras de Córdoba (Argentina). III. Revista de la Sociedad Entomológica Argentina, 58(3-4):109-127.

Rosso de Ferradás, B.; Kaisin, F. \& Bosnia, A. 1987. Seasonal variation of density and biomass of Hydracarina (Acari) in a North-Patagonian reservoir (Neuquén, Argentina) Studies on Neotropical Fauna \& Environment, Tübingen, 22(3):113-127.

Smit, H. 2002. Rheophilic water mites from southern Argentina, with the description of one new genus and three new species (Acari: Hydrachnidia). Zootaxa, Washington, 103: $1-23$.

VIETS, K. 1955. XVI. Hydrachnellae. In: Cary Gilson, H. The Percy Sladen Trust Expedition to lake Titicaca in 1937. London, Transactions Linnean Society of London. v.1, cap.3, p.249-274.

Viets, K. O. 1977. Neue und seltene wassermilben (Hydrachnellae, Acari) aus Guatemala-Teil I. Acarologia, Paris, 18(4):668-690.

1987. Die milben des süsswassers (Hydrachnellae und Halacaridae, Acari) 2:Katalog. Berlin, Verlag Paul Parey. 1012p.

Walter, D. E. \& Proctor, H. C. 1998. Predatory mites in tropical Australia: local species richness and complementarity. Biotropica, Kansas, 30(1):72-81.

WASSON, J. G. \& BARRère, B. 1999. Regionalización de la cuenca Amazónica Boliviana: Las hidro-ecoregiones de la zona Andina. Revista Boliviana de Ecología, 6:111-120. 